\title{
Graduate Student Literature Review: The milk behind the mustache: A review of milk and bone biology*
}

\author{
Brandon S. Batty† and Massimo Bionazł \\ Department of Animal and Rangeland Sciences, Oregon State University, Corvallis 97330
}

\begin{abstract}
Exploring the relationship between nutrition, skeletal development, and aging is important in maintaining bone health. Even further, understanding the complexity of skeletal homeostasis may assist in reducing the prevalence of skeletal disease, especially osteoporosis. The skeleton is unique in that it can adapt to various physical pressures, maintain shape, and remodel itself to increase integrity and strength. For decades, it was thought that increasing skeletal health was as simple as drinking three 8-oz. glasses of milk per day due to high levels of bioavailable calcium. New research into the bioactive components of milk have revealed other roles in promoting skeletal health. Milk contains various bioactive peptides, houses genetic information in milk-derived exosomes, and supplies relevant amounts of nutrients important for bone health. In this review, we discuss the basics of skeletal formation and homeostasis, dive into the potential effects of milk on the growing skeleton, and present contrasting findings.
\end{abstract}

Key words: bone, milk, osteoporosis, exosome, bioactive

\section{INTRODUCTION}

Knowledge of skeletal physiology has exponentially increased in recent years. From advances in imaging technology to unveiling the skeletal genome, the skeleton has emerged as much more than a simple, static organ. Unfortunately, like any system, the skeleton is susceptible to deterioration and disease. Therefore, understanding the intricacies of bones and how they respond to various stimuli is critical for maintaining lifelong skeletal health. At the center of skeletal health

Received February 2, 2019.

Accepted April 19, 2019.

*Submitted to the 2020 ADSA Foundation Graduate Student Literature Review Competition (Dairy Foods, MS) on April 23, 2019. †Corresponding author: brandon.batty@oregonstate.edu

$\ddagger$ Advisor (massimo.bionaz@oregonstate.edu) and development is excellent nutrition. Meeting daily nutrient requirements is important for normal development and essential for maximizing and optimizing peak bone mass. Milk, an excellent source of calcium, vitamin D (with fortification), and energy, has been thought of as the perfect food regarding its potential benefits for the skeleton. Since early Norse mythology, milk has been considered a nutrient-rich, life-giving beverage vital for maintaining health and longevity. However, concerns with its fat profile, lactose intolerance, and milk substitutes have quickly downgraded milk as an important source of nutrition. The purpose of this review is to provide a brief background on bone physiology and provide a scientific breakdown of the components in milk that may affect the skeleton.

\section{BONE FORMATION, PHYSIOLOGY, AND STRUCTURE}

\section{Bone Formation}

Bone formation occurs in 2 major modes, both of which require rapidly proliferating cells that reside in the marrow tissue and periosteal tissue. The 2 modes are intramembranous ossification and endochondral ossification.

Intramembranous ossification is responsible for the formation of the flat bones of the skeleton. This form of ossification does not use an intervening cartilage model, and neural crest-derived mesenchymal cells that migrate to sites of future bone differentiate directly into bone-committed osteoblasts (Dwek, 2010). The osteoblasts secrete a collagen-proteoglycan matrix known as osteoid that is capable of binding to calcium salts and phosphorus to construct the mineralized bone matrix known as hydroxyapatite. Although osteoblasts are usually separated from the layer of calcification, some become trapped in the osteoid matrix and terminally differentiate into osteocytes, which are mature bone cells (Schaffler et al., 2014). The transition to an osteocyte involves a dramatic shift from a cuboidal cell capable of matrix secretion and differentiation to a smallbodied cell with projecting processes for cell-to-cell 
communication. As the calcification process continues, bone spicules emanate from the osteocytes, forming a network of connected cells that are visible as lacunae and canaliculi (Schaffler et al., 2014). Directly adjacent to the developing bone is the periosteum. A fibrous sheet of connective tissue, the periosteum is tightly bound to the bone by thick fibers knows as Sharpey's fibers (Clarke, 2008). The inner surface layer of cells differentiates into osteoblasts and deposits osteoid. By this mechanism, many layers of bone are formed.

Endochondral ossification utilizes a cartilage intermediate that is subsequently replaced by bone. This process is responsible for the development of the long bones and the appendicular skeleton. First, mesenchymal stem cells become committed to the cartilage cell lineage (Gilbert, 2000). These committed cells then condense to form compact nodules and differentiate into chondrocytes. Cells near the middle of the condensations secrete a cartilage matrix consisting of type II, IX, and XI collagen as well as proteoglycans (Gilbert, 2000). Cells near the outer edge of the developing bone form the perichondrium and secrete type I collagen (Long and Ornitz, 2013). Next, the chondrocytes undergo a period of rapid proliferation that engages the linear growth of the developing bones. After rapid proliferation, the cells stop dividing, undergo hypertrophy, then apoptosis, and calcify (Dwek, 2010). In mammals, this type of ossification begins in the center of the bone and moves outward in both directions. This results in the formation of ossification centers located at both ends of the developing bone. The chondrocytes follow a pattern of hypertrophy, death, and subsequent mineralization that results in longitudinal bone growth (Gilbert, 2000; Dwek, 2010). Vascular incursion results in a source of perivascular osteoprogenitor cells and osteoclasts, both of which ensure correct skeletal development and aid in modeling and remodeling of bone throughout life (Gilbert, 2000; Dwek, 2010).

\section{Bone Mineral Profile and Milk}

Several key nutrients critical for bone health are available in one 8-oz. serving of milk. The thought that consuming dairy products improves bone health is longstanding, and many studies have sought to prove this correlation (Kalkwarf, 2007). Simply put, an increased amount of bioavailable calcium in the diet has been shown to improve bone development and health (Caroli et al., 2011; Rizzoli, 2014a). Calcium is often seen as the most important component of milk for bone health, and adequate intake is recommended during adolescence to increase peak bone mass and help prevent osteoporosis later in life (Hess et al., 2016). When mice were treated with low, adequate, and high levels of dietary calcium, low dietary calcium negatively affected bone strength and architecture and overall peak bone mass (Viguet-Carrin et al., 2014). However, the diverse blend of nutrients in milk have been shown to increase bone health and development. Besides calcium, milk contains biologically relevant amounts of phosphorus, magnesium, protein, and vitamin D (due to fortification), which all contribute to bone growth and mineral accrual (Pereira, 2014; Rizzoli, 2014b). Vitamin D is critical for maintaining calcium homeostasis and assists in the active transport of calcium across the intestinal lumen (Hess et al., 2016). Phosphorus, magnesium, and zinc are all critical to maintaining a healthy skeleton (Thorning et al., 2016). Adequate phosphorus is critical for forming the matrix of hydroxyapatite. Magnesium regulates calcium homeostasis through its interaction with parathyroid hormone. Zinc, though found in small amounts in milk, is an important cofactor for enzymes while forming part of the apatite portion of bone (Hess et al., 2016). Consuming the recommended amount of fluid milk each day, along with an adequate diet, should provide these bone-critical nutrients in sufficient amounts to reach the daily recommended intake and could help balance skeletal homeostasis and decrease the rate of bone loss and risk of osteoporosis. Table 1 lists key nutrients for bone health that are provided in one 8-oz. serving of $2 \%$ homogenized, pasteurized cow milk (USDA, 2018).

\section{Bone Microstructure}

Bone structure can be divided into 2 categories: macrostructure, which refers to the whole bone and its extrinsic properties (e.g., shape, size, and mechanical strength), and microstructure, which refers to the individual elements that make up bone. Immediately surrounding the outside of the bone is the periosteum. With the exception of articular surfaces and sesamoid bones, almost every bony surface is covered in periosteum (Dwek, 2010). The periosteum contains 2 distinct layers: a fibrous outer layer and an inner layer with osteoblastic potential (Dwek, 2010). The outer layer can further be divided into 2 layers, with the outer layer representing an inelastic, cell-poor portion that is dense with collagenous matrix, and the inner layer representing a fibroelastic layer with significant elasticity (Dwek, 2010). This inner layer of the periosteum is dense with osteogenic progenitor cells, osteoblasts, and fibroblasts that lay directly above the bone (Gilbert, 2000; Dwek, 2010). Located under the periosteum is the cortical or compact bone. Cortical bone is the dense, solid layer that surrounds the marrow cavity and is assembled by osteons, which serve as its functional units (Clarke, 2008). Together, osteons and their vascu- 
Table 1. Key nutrients available in one 8-oz. serving of cow milk compared with the daily recommended intake (DRI) for children aged 9 to $13 \mathrm{yr}^{1}$

\begin{tabular}{lccc}
\hline Nutrient & DRI & $\begin{array}{c}\text { Amount in } 8 \text { oz. } \\
\text { of } 2 \% \text { cow milk }\end{array}$ & $\begin{array}{c}\text { DRI provided in 8 oz. } \\
\text { of 2\% cow milk (\%) }\end{array}$ \\
\hline Calcium & $1,300 \mathrm{mg}$ & $293 \mathrm{mg}$ & 22.5 \\
Phosphorus & $1,250 \mathrm{mg}$ & $224 \mathrm{mg}$ & 17.9 \\
Magnesium & $240 \mathrm{mg}$ & $27 \mathrm{mg}$ & 11.2 \\
Vitamin D & $600 \mathrm{IU}$ & $120 \mathrm{IU}$ & 20.0 \\
Zinc & $8 \mathrm{mg}$ & $1.17 \mathrm{mg}$ & 14.6 \\
\hline
\end{tabular}

${ }^{1}$ Consuming the USDA-recommended three 8-oz. glasses of milk per day provides substantial quantities of these nutrients and decreases the likelihood of deficiency.

${ }^{2}$ From USDA (2018).

lature make up the Haversian canals and system (large animals only). The walls of these canals are formed of lamellae, which are concentric layers of compact bone, and lacunae, which are small pores within the bone matrix eventually populated by an osteocyte (Clarke, 2008). Connecting the lacunae are microscopic channels called canaliculi. These fluid-filled channels serve as a transport system for canicular fluid and may sense changes in load (Iwaniec and Turner, 2016), which releases antiapoptotic factors and may result in increased bone formation (Clarke, 2008; Main, 2017). These canals run parallel to each other and are connected perpendicularly by Volkmann's canal. Lamellar bone is a highly organized structure formed by remodeling woven bone - a haphazard arrangement of collagen fibers (Gilbert, 2000). Lamellar bone is stronger and provides resistance to torsion forces on the bone. Further inside the bone, particularly toward the ends of long bone, is a matrix called trabeculae, or spongy bone. This inner layer of bone has a honeycomb-like structure, affording great elasticity and flexibility. Trabecular bone is the main load-bearing bone, and its microarchitecture significantly influences bone strength (Iwaniec and Turner, 2016).

\section{Bone Biomechanics}

Bone composition and structure determine the capability of a bone to resist fracture due to extreme mechanical stress that occurs during a fall (Nanes and Kallen, 2014). Bone strength is defined as the maximum amount of force that can be tolerated by the skeleton before fracture or structural failure occurs. The properties of the bone, in addition to its structure and mineral content, allow it to absorb load by elastic deformation while resisting permanent changes in shape (Fonseca et al., 2014; Osterhoff et al., 2016). Critical measurements of bone are used to determine its capacity to resist fracture: bone mineral density, total bone volume, cortical width or thickness, and trabecular thickness, separation, and number are all variables considered when es- timating load bearing (Brandi, 2009). Trabecular bone presents 2 levels of organization that allow for load transfer. At the macroscale, trabecular bone comprises a lattice that forms the framework for a soft, cell-dense marrow that fills the intertrabecular area (Oftadeh et al., 2015). The microscale is organized in a way that allows for optimal weight distribution and forms new bone in response to increased stress (Oftadeh et al., 2015). A key attribute of bone is the ability to adapt to external stimuli by adding or removing bone. Mechanosensation by osteocytes is not fully understood, but it is generally accepted that the displacement of canicular fluid, deformed bone matrix, and graded electrical potentials are all identified by osteocytes and subsequent information is transferred to osteoblasts, or osteoclasts, or both, leading to adjustments or increases in bone mass and architecture (Boskey and Coleman, 2010; Iwaniec and Turner, 2016; Osterhoff et al., 2016). Vitamin D-fortified milk consumption may influence bone strength. High levels of dietary vitamin D have resulted in greater tibial strength and greater trabecular bone volume (Williamson et al., 2017). It is theorized that high vitamin D levels achieved with fortified milk intake are suitable for higher bone mass and osteoporosis prevention (Williamson et al., 2017). Deficiency in vitamin $\mathrm{D}$, both dietary and environmental, increases risk of bone disease and reduces bone strength and health (Hess et al., 2016).

Cortical bone mass is critical to overall bone strength and health because it makes up roughly $80 \%$ of the skeleton (Brandi, 2009). Increases in cortical porosity from excessive bone resorption and thinning of diaphyseal cortices are responsible for increased fracture risk and compromised bone integrity (Brandi, 2009; Boskey and Coleman, 2010). Bone geometry also plays an important role in the determination of bone strength. Crosssectional area, shape, and size are frequently used to evaluate potential fracture risk (Fonseca et al., 2014). Taes et al. (2010) found that men reaching peak bone mass with lower cortical area and thickness and lower trabecular density were more susceptible to fractures. 
A method of directly determining strength is a 3-point bending test, which uses a constant force applied to the bone until failure occurs (Brandi, 2009; Fonseca et al., 2014). From this, elasticity, stress, strain, and overall strength can easily be calculated. Unfortunately, this method is only suitable postmortem and with animal models, making it impossible to be used as a means for diagnosis. Another method for evaluating the mechanical properties of bone at the tissue level is microindentation. An in vivo method, microindentation is performed by inserting a small probe into the skin, displacing the periosteum, and applying force over a set number of indentation points to determine the mechanical endurance of the bone. This provides an estimate for crack resistance using a relatively noninvasive method, a valuable tool for evaluating risk of fracture in patients with osteoporosis (Diez-Perez et al., 2010). Overall, the entire composition of bone mineral density, bone geometry, and bone microarchitecture illustrates the critical variables for evaluating the strength and fracture resistance of bone.

\section{Mesenchymal Stem Cell Features and Their Involvement in Bone Repair}

Mesenchymal stem cells (MSC) are fibroblast-like cells capable of adhering to plastic under normal culture conditions and having differentiation potential into osteoblasts, adipocytes, and chondroblasts in vitro while expressing cluster of differentiation (CD) 105, CD90, CD73, and CD44 and lacking expression of CD45, CD34, CD14, CD11b, CD79, CD19, and human leukocyte antigen-DR isotype in humans (Kong et al., 2017). Originally classified as marrow stromal cells, MSC have been located throughout the body in bone marrow, adipose tissue, and the umbilical cord (Kobolak et al., 2016). Mesenchymal stem cells are multipotent cells with the capability of regenerating damaged tissue through diverse differentiation patterns stimulated by damages or other factors associated with growth and repair (Lin et al., 2017). Different lineages of MSC offer flexibility for stem cell therapy research because they each have different potentials for tissue regeneration (Kong et al., 2017). Bone regeneration, fracture healing, and osteoporosis treatment with MSC have been vigorously researched in recent decades. When a tissue injury occurs, endogenous MSC relocate to the injured area for participation in immunomodulation and tissue repair (Lin et al., 2017). Granero-Moltó et al. (2009) tracked MSC using a bioluminescence technique expressing luciferase to a fracture site and showed that MSC improve fracture healing and biomechanical properties. Bone morphogenic protein has also been identified to be critical for normal fracture healing. Bone morphogenic protein is produced by osteoprogenitor cells, MSC, osteoblasts, and chondrocytes and reside in the extracellular matrix of bone. Bone morphogenic proteins efficiently regulate the differentiation and proliferation of MSC to bone-forming osteoblasts. During fracture healing, bone morphogenic protein 2 is highly expressed and triggers a cascade of osteoblastic differentiation, chemotaxis, angiogenesis, and controlled synthesis of extracellular matrix, which are critical for normal collagen deposition (GraneroMoltó et al., 2009).

\section{Milk microRNA and Bone Development}

Micro-RNA (miRNA) are small, noncoding segments of nucleotides that function in post-transcriptional gene silencing and target messenger RNA degradation, resulting in inhibition of expression of their target genes (Nugent, 2017). After transcription occurs by RNA polymerase II, the pri-miRNA are cleaved into precursor miRNA, which are transported to the cytoplasm and processed into mature, single-stranded miRNA (Huang et al., 2017). In vitro studies have tracked various miRNA and evaluated their effect on osteogenic differentiation and osteoblastic activity. Li et al. (2015) investigated miR-26a. This specific miRNA has been negatively correlated with bone loss in osteoporotic mice with induced estrogen deficiency, conditions that simulate menopause. Through cell transfection, it was discovered that overexpression of miR-26a rescued osteogenic function of MSC derived from ovariectomized mice and increased their expression of alkaline phosphatase, an early osteogenic marker, and osteocalcin, a marker of mineralization (Li et al., 2015). Micro-RNA also regulate osteoblastic differentiation by targeting specific signaling pathways critical for bone development. One such pathway is Wnt, which induces MSC proliferation and drives differentiation toward the osteoblastic lineage (Kubota et al., 2010). Numerous other miRNA can stimulate or inhibit osteogenic pathways: miR-378 enhances osteoblast differentiation in the presence of bone morphogenic protein-2 (Huang et al., 2017), miR-335-5p is highly expressed in osteoblasts and promotes osteogenesis through downregulation of the Wnt antagonist Dickkopf-related protein 1 (Zhang et al., 2011), and high levels of miR-17-5p and miR106a inhibit mineralization and alkaline phosphatase activity (Kang and Hata, 2015). Micro-RNA can target genes and transcription factors directly related to osteogenic differentiation and may regulate bone formation. Runtrelated transcription factor 2 (Runx2) is required for commitment and differentiation of osteoblasts (Dalle Carbonare et al., 2012). This protein alone is regulated by at least 11 miRNA (Lian et al., 2012). Precise con- 
trol of Runx2 is required for proper bone formation, and overexpression does not result in increased bone formation but rather in osteopenia as increased levels of Runx2 augment the secretion of RANKL (Lian et al., 2012). Therefore, miRNA serve as a control mechanism for proper development of bone.

In addition to endogenous synthesis, miRNA can be derived from the diet. Specifically, extracellular vesicles, also known as exosomes, are highly enriched with miRNA, and virtually all the circulating miRNA are enclosed in exosomes (Turchinovich et al., 2011). The exosomes provide protection for miRNA, proteins, and lipids (Zempleni, 2017). Exosomes are absorbed by intestinal and endothelial cells by endocytosis and can accumulate in the peripheral tissues (Zempleni, 2017). However, the biological activity of miRNA derived from dietary exosomes is still controversial (Zempleni, 2017). Baier et al. (2014) found that miRNA from bovine milk exosomes are absorbed and affect gene expression in peripheral immune cells in mice and that feeding a diet devoid of cow milk-derived exosome decreased plasma concentration of miR-29b by $61 \%$ (Baier et al., 2014). Oral delivery of bovine-derived extracellular vesicles in mice was shown to increase overall osteocyte number and woven bone formation but altered mineral matrix formation, suggesting an effect on osteoblast maturation and rate of mineralization (Oliveira et al., 2016). Conversely, Snow et al. (2013) concluded that ingestion of miRNA through the diet was not a frequent mechanism for horizontal transfer of miRNA to different organisms. Overall, miRNA and exosomes could be potent sources for transfering genetic information between animals; however, further research regarding uptake and horizontal transfer of dietary miRNA is still needed.

\section{Role of Cytokine Signaling in Bone Physiology}

Osteoclasts and their precursors express a receptor from the tumor necrosis factor (TNF) family known as receptor activator of nuclear factor- $\kappa \mathrm{B}$ (RANK), which interacts with its ligand (RANKL) and activates TNF receptor-associated factors (TRAF) to stimulate osteoclastogenesis (McClung, 2007; Chen et al., 2018). Together, RANK and TRAF regulate osteoclast activity through many signaling pathways that can modulate the rate and frequency of osteoclast maturation and bone resorption (Weitzmann, 2013). The ligand RANKL is a type II homotrimeric transmembrane protein that may be secreted by osteoblasts to regulate bone resorption. By binding to its receptor, RANKL controls the differentiation, proliferation, and functionality of osteoclasts. In clinical trials where denosumab (AMG 162), a bisphosphonate antibody with high affinity for RANKL, is prescribed, there is a significant reduction in RANKL binding to RANK and a consequent decrease in urinary markers for bone turnover due to up to $80 \%$ suppression of bone turnover (Bekker et al., 2004). In a clinical trial testing the efficacy of AMG 162 on postmenopausal women with low bone mass, an increase in lumbar spine and total hip bone mineral density (frequent location of fracture) and decrease in bone turnover were observed (McClung et al., 2006). These results detail the importance of RANKL in bone resorption and potential mechanisms for treating osteoporosis therapeutically (McClung et al., 2006). In contrast to therapeutic treatment of low bone mass, osteoprotegerin (OPG) levels could hold the answer to inhibiting increased bone loss. Also known as osteoclastogenesis inhibitory factor, OPG is produced not only by osteoblasts but also by the heart, liver, and spleen (Chen et al., 2018). Osteoprotegerin is a natural inhibitor of osteoclastogenesis that acts as a decoy receptor of RANKL. When OPG and RANKL bind, RANKL is unable to bind to RANK on the immature osteoclast and stimulate the signaling cascade for the fusion of monocytes into mature osteoclasts (Boyce and Xing, 2007). Simonet et al. (1997) found that mice overexpressing OPG lacked osteoclasts, resulting in increased bone mass that led to osteopetrosis (higher than normal bone mineral density). The RANKL-RANK-OPG cytokine system was one of the most important discoveries regarding bone health and metabolism that has been made in recent decades. Inhibition of RANKL by OPG or other synthetic factors could be key in early diagnosis and slowing the progression of osteoporosis.

\section{Hormonal Regulation of Bone Metabolism}

One of the most researched areas regarding bone health is postmenopausal women and estrogen deficiency. Osteoclasts express receptors for estrogen, and inhibition of osteoclastogenesis is considered estrogen's main function in protecting against bone loss (Khosla et al., 2012). When osteoclast-specific estrogen receptor- $\alpha$ was removed, there was a substantial decrease in trabecular bone mass. Moreover, estrogen blocks activator protein-1-dependent transcription induced by RANKL and macrophage colony-stimulating factor and suppresses osteoclastic differentiation in response to RANKL binding (Srivastava et al., 2001). It has also been shown that estrogen suppresses the production of RANKL by osteoblasts and increases synthesis of its inhibitor, OPG (Khosla et al., 2012). Menopausal loss of estrogen is the main cause of imbalance in bone remodeling and increased bone turnover in women, resulting in osteoporosis (Walsh, 2018). Moreover, estrogen greatly 
increases calcium absorption and menopause causes an obligatory increase in calcium excretion, further exacerbating mineral disparity. Parathyroid hormone (PTH) is another hormone directly related to bone metabolism. Secreted by the parathyroid gland in response to low blood calcium, the 84-AA polypeptide regulates serum calcium homeostasis, renal phosphate, and calcium reabsorption and modulates bone turnover (Bellido et al., 2013). Parathyroid hormone receptors are located on osteoblasts and stimulate osteoclastogenesis by increasing secretion of RANKL and decreasing OPG release, thus increasing bone resorption and serum calcium levels (Bellido et al., 2013; Walsh, 2018). As the body ages, renal function declines and calcium absorption across the intestinal lining slows. This increases PTH levels, inducing remodeling and resorption events and eventually an imbalance in bone homeostasis, resulting in decreased bone mass and potentially osteoporosis (Walsh, 2018). In opposition to PTH is calcitonin (CT). It is well established that the effects of calcitonin on bone metabolism are through the inhibition of bone resorption (Turner et al., 2011). Calcitonin binds to its receptor on osteoclasts and disrupts the ruffled border of osteoclasts and decreases osteoclast number, therefore limiting bone resorption (Carter and Schipani, 2006). Calcitonin also protects against bone resorption by decreasing the secretory activity of osteoclasts by altering $\mathrm{Na}^{+}-\mathrm{K}^{+}$-ATPase activity and reducing acid secretion (Zaidi et al., 2002). Insulin-like growth factor 1 has various effects on the skeleton: it stimulates longitudinal bone growth, favors MSC differentiation into osteoblasts, and increases periosteal bone expansion (Walsh, 2018). Milk proteins are high in aromatic AA, which increases synthesis of IGF-1. Insulin-like growth factor 1 indirectly stimulates calcium absorption across the intestinal lumen by enhancing production of circulating calcitriol (Bonjour et al., 2009). An increase in serum IGF-1 exerts growth effects on bone mass during adulthood (Rizzoli, 2014b). Yakar et al. (2009) found that IGF-1 increases bone strength, regulates size and shape, and plays a key role in adaptation to increased mechanical stress from total body growth. In addition, Zhang et al. (2002) confirmed that IGF-1 increases the rate of mineralization, which is of critical importance during the rapid bone growth of puberty. Furthermore, IGF-1 has been shown to stimulate RANKL secretion and osteoclastogenesis and maturation, suggesting that IGF-1 is a critical regulator in bone turnover and remodeling (Locatelli and Bianchi, 2014).

\section{OSTEOPOROSIS AND BONE MINERAL DENSITY}

Bone is often regarded as a simple, static tissue that supports weight and stops growing when we reach our genetic potential for height. In reality, bone is a dynamic tissue undergoing constant remodeling (concurrent action of bone formation and bone resorption) to adjust for physical pressures, physiological stress, and overall aging (Boskey and Coleman, 2010). In addition to growing in length and volume, bone increases in overall density until peak bone mass is achieved. It is essential to reach the highest level of peak bone mass as determined by our genetics to reduce risk of osteoporotic fracture later in life (Weaver et al., 2016). As we age, and after peak bone mass has been achieved, the rate of bone formation by osteoblasts is overtaken by bone resorption by osteoclasts. When this occurs in excess, bones become weak and brittle, and there is an increased risk of fracture due to a fall. During aging, our ability to absorb minerals vital for bone health decreases. As this occurs, we must increase intake to balance the loss of absorption. However, reaching the daily required amounts of these minerals may be difficult with a typical Western diet, and deficiency is common (Nordin, 1997). The clinical term for this decrease in bone mass and integrity is osteoporosis. Defined quantitively, osteoporosis is diagnosed when body bone mineral density is at least 2.5 SD below a reference range of healthy individuals (Eastell, 2017). Considered a silent disease, osteoporosis affects approximately 35 million Americans and results in $\$ 17$ to $\$ 20$ billion in health care costs annually due to osteoporosis-related injuries (Nanes and Kallen, 2014). Diagnosis of osteoporosis can be achieved through dual-energy X-ray absorptiometry, the gold standard for measuring bone mineral density that provides a simple, noninvasive method of pinpointing individuals who may be at an increased risk for fracture (Nanes and Kallen, 2014; Wilkinson et al., 2015). Dual-energy X-ray absorptiometry uses high- and low-energy photons to differentiate between hard and soft tissue. Photons are absorbed by the bone, and the remaining photons not absorbed are used to determine bone mineral density; higher absorption means greater bone mineral density (Wilkinson et al., 2015). Although dual-energy X-ray absorptiometry is a very powerful tool in osteoporosis diagnosis, it is only a 2-dimensional analysis of a 3-dimensional problem (Wilkinson et al., 2015). Bone has a diverse role in the body that is somewhat paradoxical. It must provide support and protection and serve as a reserve for calcium and other minerals. If bone is too dense, it will not have the flexibility to absorb mechanical load and will fail; if bone is too soft, it will break under stress and have no physical capacity to aid in locomotion. This creates a need not only to assess bone mineral density for prevention and diagnosis of osteoporosis but also to evaluate bone quality and its microarchitecture (Yuan et al., 2016). 


\section{EFFECT OF MILK ON BONE HEALTH: BEYOND CALCIUM AND VITAMIN D}

\section{Bioactive Protein-Derived Compounds in Milk and Their Potential Effects on Bone}

Milk is the first source of nutrients for mammals and contains critical amounts of nutrients vital for growth and development (Pereira, 2014). Uniquely for humans, milk consumption continues past the weaning stage and may continue throughout one's entire life. Considered an important factor of a healthy diet, milk contains a diverse source of nutrients and various bioactive compounds (Hsieh et al., 2015). The protein fraction of milk is receiving further attention regarding its application for improving bone health. The protein fraction of milk comprises 2 main portions, approximately $80 \%$ being casein proteins and $20 \%$ being whey proteins. Further classification of casein proteins have split the group into $\alpha-, \beta-$, and $\kappa-\mathrm{CN}$, whereas the whey portion contains the major constituents $\beta-\mathrm{LG}, \alpha-\mathrm{LA}$, and various other minor proteins (Mohanty et al., 2016). When ingested, milk proteins undergo enzymatic digestion that yields milk peptides. These milk-derived peptides have bioactive properties (Mohanty et al., 2016). Such peptides have antimicrobial, immunomodulatory, antioxidant, bone-forming, and various other properties that suggest that ingestion of milk is beneficial nutritionally and could serve as a nutraceutical (Mohanty et al., 2016). A study by Toba et al. (2001) followed 30 adult men receiving a beverage containing $300 \mathrm{mg}$ of milk basic protein daily for $16 \mathrm{~d}$. Serum osteocalcin, a product of osteoblast activity, significantly increased in the treatment group, suggesting the increased activity of bone formation. In the same study, urinary crosslinked $N$-telopeptides of type- 1 collagen, a measure of bone resorption, was significantly decreased. Thus, supplementation of milk basic protein increased bone formation and decreased bone resorption (Toba et al., 2001). An in vitro study evaluating concentrated bovine milk whey active proteins determined that these proteins can promote osteoblastic differentiation and mineralization under normal human culture conditions (Tsuji-Naito and Jack, 2012). Physiological digestion of the casein protein portion of milk yields various peptides with effects on bone metabolism (Tulipano et al., 2010). Casein phosphopeptides (CPP) may enhance calcium solubility and bone calcification. The ability of CPP to reduce precipitation of calcium likely results in increased intestinal calcium absorption. The increase in interstitial calcium may provide preosteoblasts with a differentiation signal to begin the descent to mature osteoblasts and begin depositing inorganic matrix for calcification. Mora-Gutierrez et al. (2007) evaluated calcium absorption in growing mice given different ratios of CPP and evaluated calcium absorption and skeletal deposition. Using ${ }^{45} \mathrm{Ca}$, it was found that all ratio of CPP increased plasma calcium and calcium uptake by the femur, suggesting an increase in osteogenesis and bone formation (Mora-Gutierrez et al., 2007). Overall, CPP have the potential to increase intestinal calcium absorption, directly affect osteoblast-like cell growth, and result in greater deposition of calcium in bone extracellular matrix (Tulipano et al., 2010). Using a rodent model for oral administration of lactoferrin, it was concluded that lactoferrin can act as a preserving agent for bone mass and microarchitecture in ovariectomized mice. Lactoferrin can offset estrogen-dependent bone loss and deterioration (Guo et al., 2009). Work presented by Bharadwaj et al. (2009) found that supplementation of milk ribonuclease-enriched lactoferrin significantly reduced osteoclastic activity, increased bone formation, and rescued bone turnover rates in 38 healthy postmenopausal women. Contributing to the research of milk as a functional food, bioactive peptides may be another critical piece in the puzzle against disease. Specifically, bioactive peptides from milk can protect against bone loss both in vitro and in vivo, and their effect on the expression of genes regarding bone formation may confirm their role as a nutraceutical (Mohanty et al., 2016).

\section{Conflicting Literature}

Although milk has been proven to be beneficial for bone health, some studies suggest otherwise. Michaëlsson et al. (2014) evaluated the relationship between high milk consumption (3+8-oz. glasses/d), mortality, and fracture risk. High intake was associated with increased mortality in men and women and with an increased risk of fracture in women. As this study is observational with the possibility of confounding variables, results should be interpreted cautiously (Michaëlsson et al., 2014). Even so, a 22-yr prospective follow-up of the Nurses' Health Study and the Health Professionals Follow-Up study evaluated the risk of hip fracture at 50 yr or older associated with milk intake during the ages of 13 to 18 yr. An additional glass of milk per day was associated with significantly higher hip fracture risk in men, whereas milk consumption had no effect on hip fracture risk in women (Feskanich et al., 2014). The latest systematic review and meta-analysis revealed inconsistent and unreliable evidence for the association of milk consumption and risk of hip fracture (Bian et al., 2018). Further research into the direct effect of milk on fracture risk in both the short and long term is needed. 


\section{CONCLUSIONS}

The skeleton is a complex and intricate organ that is highly regulated by many pathways, stimuli, and hormones. Along with this comes modifiable factors that play an important role in achieving peak bone mass, preventing osteoporosis, and maintaining a life free from physical pain caused by a deteriorating skeleton. Focusing on optimal nutrition and instilling a healthy lifestyle have proven to provide the skeleton with the building blocks it needs to stay strong and useful. Milk can assist the development of the skeleton and can be a valuable part of the diet. However, further research into the direct effect of milk on the skeleton is needed. Specifically, how milk affects bone strength, growth, and mineral accrual during the peripubertal period as well as how milk and its bioactive compounds may affect stem cell proliferation and differentiation and expression patterns of genes related to bone turnover will be important gaps to fill in future years.

\section{REFERENCES}

Baier, S. R., C. Nguyen, F. Xie, J. R. Wood, and J. Zempleni. 2014. MicroRNAs are absorbed in biologically meaningful amounts from nutritionally relevant doses of cow milk and affect gene expression in peripheral blood mononuclear cells, HEK-293 kidney cell cultures, and mouse livers. J. Nutr. 144:1495-1500. https://doi.org/ 10.3945/jn.114.196436.

Bekker, P. J., D. L. Holloway, A. S. Rasmussen, R. Murphy, S. W. Martin, P. T. Leese, G. B. Holmes, C. R. Dunstan, and A. M. DePaoli. 2004. A single-dose placebo-controlled study of AMG 162, a fully human monoclonal antibody to RANKL, in postmenopausal women. J. Bone Miner. Res. 19:1059-1066. https://doi.org/10 .1359/JBMR.040305.

Bellido, T., V. Saini, and P. D. Pajevic. 2013. Effects of PTH on osteocyte function. Bone 54:250-257. https://doi.org/10.1016/j.bone 2012.09.016

Bharadwaj, S., A. G. T. Naidu, G. V. Betageri, N. V. Prasadarao, and A. S. Naidu. 2009. Milk ribonuclease-enriched lactoferrin induces positive effects on bone turnover markers in postmenopausal women. Osteoporos. Int. 20:1603-1611. https://doi.org/10.1007/ s00198-009-0839-8.

Bian, S., J. Hu, K. Zhang, Y. Wang, M. Yu, and J. Ma. 2018. Dairy product consumption and risk of hip fracture: A systematic review and meta-analysis. BMC Public Health 18. https://doi.org/ 10.1186/s12889-018-5041-5.

Bonjour, J.-P., T. Chevalley, S. Ferrari, and R. Rizzoli. 2009. The importance and relevance of peak bone mass in the prevalence of osteoporosis. Salud Publica Mex. 51(Suppl. 1):S5-S17. https://doi .org/10.1590/S0036-36342009000700004.

Boskey, A. L., and R. Coleman. 2010. Aging and bone. J. Dent. Res. 89:1333-1348. https://doi.org/10.1177/0022034510377791.

Boyce, B. F., and L. Xing. 2007. Biology of RANK, RANKL, and osteoprotegerin. Arthritis Res. Ther. 9(Suppl. 1):S1. https://doi .org/10.1186/ar2165.

Brandi, M. L. 2009. Microarchitecture, the key to bone quality. Rheumatology (Oxford) 48:iv3-iv8. https://doi.org/10.1093/ rheumatology/kep273.

Caroli, A., A. Poli, D. Ricotta, G. Banfi, and D. Cocchi. 2011. Invited review: Dairy intake and bone health: A viewpoint from the state of the art. J. Dairy Sci. 94:5249-5262. https://doi.org/10.3168/jds .2011-4578.
Carter, P. H., and E. Schipani. 2006. The roles of parathyroid hormone and calcitonin in bone remodeling: Prospects for novel therapeutics. Endocr. Metab. Immune Disord. 6:59-76. https://doi.org/10 $.2174 / 187153006776056666$.

Chen, X., Z. Wang, N. Duan, G. Zhu, E. M. Schwarz, and C. Xie. 2018. Osteoblast-osteoclast interactions. Connect. Tissue Res. 59:99-107. https://doi.org/10.1080/03008207.2017.1290085.

Clarke, B. 2008. Normal bone anatomy and physiology. Clin. J. Am. Soc. Nephrol. 3:S131-S139. https://doi.org/10.2215/CJN .04151206 .

Dalle Carbonare, L., G. Innamorati, and M. T. Valenti. 2012. Transcription factor Runx2 and its application to bone tissue engineering. Stem Cell Rev. 8:891-897. https://doi.org/10.1007/s12015 -011-9337-4.

Diez-Perez, A., R. Güerri, X. Nogues, E. Cáceres, M. J. Peña, L. Mellibovsky, C. Randall, D. Bridges, J. C. Weaver, A. Proctor, D. Brimer, K. J. Koester, R. O. Ritchie, and P. K. Hansma. 2010. Microindentation for in vivo measurement of bone tissue mechanical properties in humans. J. Bone Miner. Res. 25:1877-1885. https: //doi.org/10.1002/jbmr.73.

Dwek, J. R. 2010. The periosteum: What is it, where is it, and what mimics it in its absence? Skeletal Radiol. 39:319-323. https://doi .org/10.1007/s00256-009-0849-9.

Eastell, R. 2017. Prevention and management of osteoporosis. Medicine (Baltimore) 45:565-569. https://doi.org/10.1016/j.mpmed 2017.06.004.

Feskanich, D., H. A. Bischoff-Ferrari, L. Frazier, and W. C. Willett. 2014. Milk consumption during teenage years and risk of hip fractures in older adults. JAMA Pediatr. 168:54-60. https://doi.org/ 10.1001/jamapediatrics.2013.3821

Fonseca, H., D. Moreira-Gonçalves, H.-J. A. Coriolano, and J. A. Duarte. 2014. Bone quality: The determinants of bone strength and fragility. Sports Med. 44:37-53. https://doi.org/10.1007/s40279 -013-0100-7.

Gilbert, S. F. 2000. Developmental Biology. 6th ed. Osteogenesis: The Development of Bones. https://www.ncbi.nlm.nih.gov/books/ NBK10056/. Sinauer Associates, Sunderland, MA.

Granero-Moltó, F., J. A. Weis, M. I. Miga, B. Landis, T. J. Myers, L. O'Rear, L. Longobardi, E. D. Jansen, D. P. Mortlock, and A. Spagnoli. 2009. Regenerative effects of transplanted mesenchymal stem cells in fracture healing. Stem Cells 27:1887-1898. https:// doi.org/10.1002/stem.103.

Guo, H. Y., L. Jiang, S. A. Ibrahim, L. Zhang, H. Zhang, M. Zhang, and F. Z. Ren. 2009. Orally administered lactoferrin preserves bone mass and microarchitecture in ovariectomized rats. J. Nutr. 139:958-964. https://doi.org/10.3945/jn.108.100586.

Hess, J. M., S. S. Jonnalagadda, and J. L. Slavin. 2016. Dairy foods: Current evidence of their effects on bone, cardiometabolic, cognitive, and digestive health. Compr. Rev. Food Sci. Food Saf. 15:251-268. https://doi.org/10.1111/1541-4337.12183.

Hsieh, C.-C., B. Hernández-Ledesma, S. Fernández-Tomé, V. Weinborn, D. Barile, and J. M. L. N. de Moura Bell. 2015. Milk proteins, peptides, and oligosaccharides: Effects against the 21st century disorders. BioMed Res. Int. 2015. https://doi.org/10.1155/ $2015 / 146840$.

Huang, C., J. Geng, and S. Jiang. 2017. MicroRNAs in regulation of osteogenic differentiation of mesenchymal stem cells. Cell Tissue Res. 368:229-238. https://doi.org/10.1007/s00441-016-2462-2.

Iwaniec, U. T., and R. T. Turner. 2016. Influence of body weight on bone mass, architecture and turnover. J. Endocrinol. 230:R115R130. https://doi.org/10.1530/JOE-16-0089.

Kalkwarf, H. J. 2007. Childhood and adolescent milk intake and adult bone health. Int. Congr. Ser. 1297:39-49. https://doi.org/10.1016/ j.ics.2006.08.008.

Kang, H., and A. Hata. 2015. The role of microRNAs in cell fate determination of mesenchymal stem cells: Balancing adipogenesis and osteogenesis. BMB Rep. 48:319-323. https://doi.org/10.5483/ BMBRep.2015.48.6.206.

Khosla, S., M. J. Oursler, and D. G. Monroe. 2012. Estrogen and the skeleton. Trends Endocrinol. Metab. 23:576-581. https://doi.org/ 10.1016/j.tem.2012.03.008. 
Kobolak, J., A. Dinnyes, A. Memic, A. Khademhosseini, and A. Mobasheri. 2016. Mesenchymal stem cells: Identification, phenotypic characterization, biological properties and potential for regenerative medicine through biomaterial micro-engineering of their niche. Methods 99:62-68. https://doi.org/10.1016/j.ymeth.2015.09.016.

Kong, L., L.-Z. Zheng, L. Qin, and K. K. W. Ho. 2017. Role of mesenchymal stem cells in osteoarthritis treatment. J. Orthop. Translat. 9:89-103. https://doi.org/10.1016/j.jot.2017.03.006.

Kubota, T., T. Michigami, and K. Ozono. 2010. Wnt signaling in bone. Clin. Pediatr. Endocrinol. 19:49-56. https://doi.org/10 $.1297 /$ cpe.19.49.

Li, Y., L. Fan, J. Hu, L. Zhang, L. Liao, S. Liu, D. Wu, P. Yang, L. Shen, J. Chen, and Y. Jin. 2015. MiR-26a rescues bone regeneration deficiency of mesenchymal stem cells derived from osteoporotic mice. Mol. Ther. 23:1349-1357. https://doi.org/10.1038/mt 2015.101.

Lian, J. B., G. S. Stein, A. J. van Wijnen, J. L. Stein, M. Q. Hassan, T. Gaur, and Y. Zhang. 2012. MicroRNA control of bone formation and homeostasis. Nat. Rev. Endocrinol. 8:212-227. https:// doi.org/10.1038/nrendo.2011.234.

Lin, W., L. Xu, S. Zwingenberger, E. Gibon, S. B. Goodman, and G. Li. 2017. Mesenchymal stem cells homing to improve bone healing. J. Orthop. Translat. 9:19-27. https://doi.org/10.1016/j.jot.2017 .03 .002 .

Locatelli, V., and V. E. Bianchi. 2014. Effect of GH/IGF-1 on bone metabolism and osteoporosis. Int. J. Endocrinol. 2014:235060.

Long, F., and D. M. Ornitz. 2013. Development of the endochondral skeleton. Cold Spring Harb. Perspect. Biol. 5:a008334. https://doi .org/10.1101/cshperspect.a008334.

Main, R. P. 2017. Osteocytes and the bone lacunar-canalicular system: Insights into bone biology and skeletal function using bone tissue microstructure. Int. J. Paleopathol. 18:44-46. https://doi.org/10 .1016/j.ijpp.2017.05.002.

McClung, M. 2007. Role of RANKL inhibition in osteoporosis. Arthritis Res. Ther. 9:S3. https://doi.org/10.1186/ar2167.

McClung, M. R., E. M. Lewiecki, S. B. Cohen, M. A. Bolognese, G. C. Woodson, A. H. Moffett, M. Peacock, P. D. Miller, S. N. Lederman, C. H. Chesnut, D. Lain, A. J. Kivitz, D. L. Holloway, C. Zhang, M. C. Peterson, and P. J. Bekker. 2006. Denosumab in postmenopausal women with low bone mineral density. N. Engl. J. Med. 354:821-831. https://doi.org/10.1056/NEJMoa044459.

Michaëlsson, K., A. Wolk, S. Langenskiöld, S. Basu, E. Warensjö Lemming, H. Melhus, and L. Byberg. 2014. Milk intake and risk of mortality and fractures in women and men: Cohort studies. BMJ 349:g6015. https://doi.org/10.1136/bmj.g6015.

Mohanty, D. P., S. Mohapatra, S. Misra, and P. S. Sahu. 2016. Milk derived bioactive peptides and their impact on human health-A review. Saudi J. Biol. Sci. 23:577-583. https://doi.org/10.1016/j .sjbs.2015.06.005.

Mora-Gutierrez, A., H. M. Farrell, R. Attaie, V. J McWhinney, and C. Wang. 2007. Influence of bovine and caprine casein phosphopeptides differing in alphas1-casein content in determining the absorption of calcium from bovine and caprine calcium-fortified milks in rats. J. Dairy Res. 74:356-366.

Nanes, M. S., and C. B. Kallen. 2014. Osteoporosis. Semin. Nucl. Med. 44:439-450. https://doi.org/10.1053/j.semnuclmed.2014.06.006.

Nordin, B. E. C. 1997. Calcium and osteoporosis. Nutrition 13:664686. https://doi.org/10.1016/S0899-9007(97)83011-0.

Nugent, M. 2017. MicroRNAs and fracture healing. Calcif. Tissue Int. 101:355-361. https://doi.org/10.1007/s00223-017-0296-x.

Oftadeh, R., M. Perez-Viloria, J. C. Villa-Camacho, A. Vaziri, and A. Nazarian. 2015. Biomechanics and mechanobiology of trabecular bone: A review. J. Biomech. Eng. 137. https://doi.org/10.1115/1 4029176 .

Oliveira, M. C., O. J. Arntz, E. N. Blaney Davidson, P. L. E. M. van Lent, M. I. Koenders, P. M. van der Kraan, W. B. van den Berg, A. V. M. Ferreira, and F. A. J. van de Loo. 2016. Milk extracellular vesicles accelerate osteoblastogenesis but impair bone matrix formation. J. Nutr. Biochem. 30:74-84. https://doi.org/10.1016/j .jnutbio.2015.11.017.
Osterhoff, G., E. F. Morgan, S. J. Shefelbine, L. Karim, L. M. McNamara, and P. Augat. 2016. Bone mechanical properties and changes with osteoporosis. Injury 47:S11-S20. https://doi.org/10.1016/ S0020-1383(16)47003-8.

Pereira, P. C. 2014. Milk nutritional composition and its role in human health. Nutrition 30:619-627. https://doi.org/10.1016/j.nut .2013.10.011.

Rizzoli, R. 2014a. Nutritional aspects of bone health. Best Pract. Res. Clin. Endocrinol. Metab. 28:795-808. https://doi.org/10.1016/j .beem.2014.08.003.

Rizzoli, R. 2014b. Dairy products, yogurts, and bone health. Am. J. Clin. Nutr. 99:1256S-1262S. https://doi.org/10.3945/ajcn.113 .073056 .

Schaffler, M. B., W.-Y. Cheung, R. Majeska, and O. Kennedy. 2014. Osteocytes: Master orchestrators of bone. Calcif. Tissue Int. 94:524. https://doi.org/10.1007/s00223-013-9790-y.

Simonet, W. S., D. L. Lacey, C. R. Dunstan, M. Kelley, M. S. Chang, R. Lüthy, H. Q. Nguyen, S. Wooden, L. Bennett, T. Boone, G. Shimamoto, M. DeRose, R. Elliott, A. Colombero, H. L. Tan, G. Trail, J. Sullivan, E. Davy, N. Bucay, L. Renshaw-Gegg, T. M. Hughes, D. Hill, W. Pattison, P. Campbell, S. Sander, G. Van, J. Tarpley, P. Derby, R. Lee, and W. J. Boyle. 1997. Osteoprotegerin: A novel secreted protein involved in the regulation of bone density. Cell 89:309-319.

Snow, J. W., A. E. Hale, S. K. Isaacs, A. L. Baggish, and S. Y. Chan. 2013. Ineffective delivery of diet-derived microRNAs to recipient animal organisms. RNA Biol. 10:1107-1116. https://doi.org/10 $.4161 /$ rna.24909.

Srivastava, S., G. Toraldo, M. N. Weitzmann, S. Cenci, F. P. Ross, and R. Pacifici. 2001. Estrogen decreases osteoclast formation by down-regulating receptor activator of NF- $\mathrm{BB}$ ligand (RANKL)induced JNK activation. J. Biol. Chem. 276:8836-8840. https:// doi.org/10.1074/jbc.M010764200.

Taes, Y., B. Lapauw, G. Vanbillemont, D. De Bacquer, S. Goemaere, H. Zmierczak, and J. M. Kaufman. 2010. Prevalent fractures are related to cortical bone geometry in young healthy men at age of peak bone mass. J. Bone Miner. Res. 25:1433-1440. https://doi .org/10.1002/jbmr.17.

Thorning, T. K., A. Raben, T. Tholstrup, S. S. Soedamah-Muthu, I. Givens, and A. Astrup. 2016. Milk and dairy products: Good or bad for human health? An assessment of the totality of scientific evidence. Food Nutr. Res. 60:32527. https://doi.org/10.3402/fnr .v60.32527.

Toba, Y., Y. Takada, Y. Matsuoka, Y. Morita, M. Motouri, T. Hirai, T. Suguri, S. Aoe, H. Kawakami, M. Kumegawa, A. Takeuchi, and A. Itabashi. 2001. Milk basic protein promotes bone formation and suppresses bone resorption in healthy adult men. Biosci. Biotechnol. Biochem. 65:1353-1357. https://doi.org/10.1271/bbb.65.1353.

Tsuji-Naito, K., and R. W. Jack. 2012. Concentrated bovine milk whey active proteins facilitate osteogenesis through activation of the JNK-ATF4 pathway. Biosci. Biotechnol. Biochem. 76:11501154. https://doi.org/10.1271/bbb.110990.

Tulipano, G., O. Bulgari, S. Chessa, A. Nardone, D. Cocchi, and A. Caroli. 2010. Direct effects of casein phosphopeptides on growth and differentiation of in vitro cultured osteoblastic cells (MC3T3E1). Regul. Pept. 160:168-174. https://doi.org/10.1016/j.regpep .2009.11.018.

Turchinovich, A., L. Weiz, A. Langheinz, and B. Burwinkel. 2011. Characterization of extracellular circulating microRNA. Nucleic Acids Res. 39:7223-7233. https://doi.org/10.1093/nar/gkr254.

Turner, A. G., F. Tjahyono, W. S. M. Chiu, J. Skinner, R. Sawyer, A. J. Moore, H. A. Morris, D. M. Findlay, J. D. Zajac, and R. A. Davey. 2011. The role of the calcitonin receptor in protecting against induced hypercalcemia is mediated via its actions in osteoclasts to inhibit bone resorption. Bone 48:354-361. https://doi .org/10.1016/j.bone.2010.09.013.

USDA. 2018. Milk, reduced fat, fluid, $2 \%$ milkfat, with added vitamin A and vitamin D. Accessed Mar. 3, 2019. https://ndb.nal.usda .gov/ndb/foods/show/72?n1=\%7BQv\%3D1\%7D\&fgcd=\&man $=\&$ ffacet $=\&$ count $=\& \max =50 \&$ sort $=$ default $\&$ qlookup $=$ milk +2 
$\% 25+$ fluid + cow $\&$ offset $=\&$ format $=$ Full $\&$ new $=\&$ measureby $=\& \mathrm{Qv}$ $=1 \& \mathrm{ds}=$ Standard + Reference\& $\mathrm{qt}=\& \mathrm{qp}=\& \mathrm{q} a=\& \mathrm{qn}=\& \mathrm{q}=\& \mathrm{ing}=$.

Viguet-Carrin, S., M. Hoppler, F. Membrez Scalfo, J. Vuichoud, M. Vigo, E. A. Offord, and P. Ammann. 2014. Peak bone strength is influenced by calcium intake in growing rats. Bone 68:85-91. https: //doi.org/10.1016/j.bone.2014.07.029.

Walsh, J. S. 2018. Normal bone physiology, remodelling and its hormonal regulation. Surgery 36:1-6. https://doi.org/10.1016/j .mpsur.2017.10.006.

Weaver, C. M., C. M. Gordon, K. F. Janz, H. J. Kalkwarf, J. M. Lappe, R. Lewis, M. O'Karma, T. C. Wallace, and B. S. Zemel. 2016. The National Osteoporosis Foundation's position statement on peak bone mass development and lifestyle factors: A systematic review and implementation recommendations. Osteoporos. Int. 27:1281-1386. https://doi.org/10.1007/s00198-015-3440-3.

Weitzmann, M. N. 2013. The role of inflammatory cytokines, the RANKL/OPG axis, and the immunoskeletal interface in physiological bone turnover and osteoporosis. Scientifica 2013:125705.

Wilkinson, J. M., R. M. Morris, M. A. Martin-Fernandez, J. M. Pozo, A. F. Frangi, M. Maheson, and L. Yang. 2015. Use of high resolution dual-energy X-ray absorptiometry-region free analysis (DXARFA) to detect local periprosthetic bone remodeling events. J. Orthop. Res. 33:712-716. https://doi.org/10.1002/jor.22823.

Williamson, L., A. Hayes, E. D. Hanson, P. Pivonka, N. A. Sims, and J. H. Gooi. 2017. High dose dietary vitamin $\mathrm{D}_{3}$ increases bone mass and strength in mice. Bone Rep. 6:44-50. https://doi.org/10 $.1016 /$ j.bonr.2017.02.001.
Yakar, S., E. Canalis, H. Sun, W. Mejia, Y. Kawashima, P. Nasser, H.W. Courtland, V. Williams, M. Bouxsein, C. Rosen, and K. J. Jepsen. 2009. Serum IGF-1 determines skeletal strength by regulating subperiosteal expansion and trait interactions. J. Bone Miner. Res. 24:1481-1492. https://doi.org/10.1359/jbmr.090226.

Yuan, Y., X. Chen, L. Zhang, J. Wu, J. Guo, D. Zou, B. Chen, Z. Sun, C. Shen, and J. Zou. 2016. The roles of exercise in bone remodeling and in prevention and treatment of osteoporosis. Prog. Biophys. Mol. Biol. 122:122-130. https://doi.org/10.1016/j.pbiomolbio 2015.11.005.

Zaidi, M., A. M. Inzerillo, B. S. Moonga, P. J. R. Bevis, and C. L. H. Huang. 2002. Forty years of calcitonin-Where are we now? A tribute to the work of Iain Macintyre, FRS. Bone 30:655-663. https://doi.org/10.1016/S8756-3282(02)00688-9.

Zempleni, J. 2017. Milk exosomes: Beyond dietary microRNAs. Genes Nutr. 12. https://doi.org/10.1186/s12263-017-0562-6.

Zhang, J., Q. Tu, L. F. Bonewald, X. He, G. Stein, J. Lian, and J. Chen. 2011. Effects of miR-335-5p in modulating osteogenic differentiation by specifically downregulating Wnt antagonist DKK1. J. Bone Miner. Res. 26. https://doi.org/10.1002/jbmr.377.

Zhang, M., S. Xuan, M. L. Bouxsein, D. von Stechow, N. Akeno, M. C. Faugere, H. Malluche, G. Zhao, C. J. Rosen, A. Efstratiadis, and T. L. Clemens. 2002. Osteoblast-specific knockout of the insulinlike growth factor (IGF) receptor gene reveals an essential role of IGF signaling in bone matrix mineralization. J. Biol. Chem. 277:44005-44012. https://doi.org/10.1074/jbc.M208265200. 